\title{
$A S P P 2$ expression predicts the prognosis of patients with hepatocellular carcinoma after transcatheter arterial chemoembolization
}

\author{
JIAREN MAO, ZHONGJUN TAN, XIAOQI PAN and FEIJIAN MENG \\ Department of Radiology, The People's Hospital of Danyang, Danyang, Jiangsu 212300, P.R. China
}

Received June 10, 2019; Accepted December 19, 2019

DOI: $10.3892 /$ etm.2021.9828

\begin{abstract}
Transcatheter arterial chemoembolization (TACE) induces ischemia-hypoxia and local chemotherapy-induced cytotoxicity which destroys cancerous cells. However, some patients do not respond to TACE. The causes for such a lack of response remain unclear. Recent studies have revealed that self-regulation of apoptosis-stimulating p53 protein 2 (ASPP2) may play an important role in promoting cell survival under hypoxic conditions as well as chemotherapy resistance via autophagy in various types of cancer. We measured the expression of ASPP2, autophagy-related proteins and apoptotic proteins by western blot assays. Multivariate logistic regression analysis was used to identify the independent risk factor. The present study found that ASPP2 expression was negatively correlated with that of BECN-1 (Beclin-1) in hepatocellular carcinoma (HCC) tissues. The expression of ASPP-1 was lower while that of Beclin-1 was higher in patients who underwent recurrence of HCC following TACE, than in those who do not undergo such a relapse. ASPP2 expression was also lower in cancerous tissues subjected to TACE, compared with that of directly resected cancerous tissue. The expression of LC3-II was also higher in patients with post-operative recurrence of $\mathrm{HCC}$ than in those without relapse. In vitro experiments showed that administration of an autophagy inhibitor, together with hypoxia activation and 5-FU treatment, promoted apoptosis in HepG2 liver cancer cells and primary HCC cells. Multivariate logistic regression analysis revealed that ASPP2 expression in cancer tissue following TACE is an independent risk factor for HCC recurrence as well as overall survival. Higher levels of ASPP2 expression were notably associated with higher objective responses evaluated via mRECIST. Thus, patients with resectable HCC showing high levels of ASPP2 expression may benefit from neoadjuvant TACE prior to resection.
\end{abstract}

Correspondence to: Dr Jiaren Mao, Department of Radiology, The People's Hospital of Danyang, 2 Xinmin West Road, Danyang, Jiangsu 212300, P.R. China

E-mail: iiiaty@163.com

Key words: transcatheter arterial chemoembolization, ASPP2, Beclin-1, hepatocellular carcinoma
Our study provided a novel biomarker for HCC prognosis following TACE, based on cell survival mechanisms related to autophagy.

\section{Introduction}

Transcatheter arterial chemoembolization (TACE) is a selective therapeutic strategy which is optimal for hepatocellular carcinoma (HCC) patients who are not indicated for direct surgical resection, such as those with a single cancerous nodule (1). The value of preoperative adjuvant TACE as an optional therapeutic strategy to improve prognoses for advanced HCC patients with resectable HCC is a subject of controversy $(2,3)$. Some studies have shown that preoperative TACE does not improve the condition of patients with resectable $\mathrm{HCC}$, and that these patients do not benefit from preoperative TACE followed by surgical resection $(4,5)$. The findings demonstrated that TACE is not beneficial in advanced HCC patients, and that preoperative TACE leads to worsen liver function, which is associated with higher mortality. However, another study demonstrated that patients showing an objective response to preoperative TACE may have a better prognosis following surgical resection, than non-responders (6). A study from Chinese Taiwan evidenced the association of preoperative TACE with improved long-term outcomes for patients, despite not having an effect on disease-free survival (DFS) or overall survival (OS) (7). Majno et al (8) reported that $62 \%$ of HCC patients, who were initially deemed unresectable, experienced a downstaging of their tumors due to necrosis induced by TACE, followed by a significant improvement in DFS after liver resection. For some patients with unresectable tumors, TACE administration further improved their suitability for resection. Therefore, objective response and the presence of HCC downstaging following preoperative TACE, may be considered as a predictor of improved prognosis following neoadjuvant TACE and liver resection.

TACE may cause changes in various markers, such as stemness markers, markers of hypoxia and tumor stromal markers $(9,10)$. Furthermore, Xu et al (11) reported that administration of TACE prior to surgery may activate hypoxia-inducible factor $1 \alpha(\mathrm{HIF}-1 \alpha)$, which is responsible for a poor prognosis of HCC. Increased expression of these molecular markers may biologically promote aggressive HCC. Autophagy may protect cancerous cells from cell death 
due to adverse conditions, such as hypoxia, starvation, and chemotherapy-induced cellular apoptosis (12-15). Therefore, evaluation of autophagy in cancer tissue following TACE may be valuable for assessing the resistance to hypoxia and chemotherapy and to predict therapeutic objective response as well.

Apoptosis-stimulating p53 protein 2 (ASPP2) may stimulate the apoptosis of cancer cells with, or without, exerting a pro-apoptotic function via p53 signaling. It is defined as a tumor suppressor which promotes apoptosis and inhibits cell growth $(16,17)$. Furthermore, in HCC, XIAP expression is reduced by ASPP2, which facilitates sensitivity to chemotherapeutics in a p53-free manner (18). A recent study reported that, in response to oxidative stress, Beclin-1-mediated autophagy is pivotal to the survival of pediatric leukemia cells (19). In addition, administration of exemestane was found to result in increased Beclin-1 and LC3 in cancer cells, where stromal levels of Beclin-1 are associated with enhanced proliferation of cancer cells and a lower sensitivity to neoadjuvant endocrine treatment $(20,21)$. Therefore, under stress conditions, autophagy in cancer tissues may contribute to cell survival and cell proliferation.

The present study investigated the expression of ASPP2 and the autophagy marker, Beclin-1, in carcinoma and para-carcinoma tissues of HCC patients following TACE. Results of in vitro experiments indicated that inhibition of autophagy promoted cell apoptosis in HepG2 liver cancer cells under adverse conditions. We provide a novel biomarker for predicting the objective response and clinical prognosis following TACE based on cell survival via autophagy under hypoxia conditions and local chemotherapy.

\section{Materials and methods}

Patient enrollment. This observational study was approved by the Medical Ethics Committee of the People's Hospital of Danyang (Danyang, Jiangsu). Written consent for collection of the tissue samples was acquired from all patients prior to surgery. HCC samples were collected directly from resected tissues of 165 , and 437 patients subjected to TACE in combination with liver resection, including unresectable patients who needed tumor staging therapy via preoperative TACE, and resectable patients who received preoperative neoadjuvant TACE. Samples were collected from primary HCC patients between January 2015 and February 2018 at the Department of Radiology, People's Hospital of Danyang. In the present study, patient enrollment was based on the following criteria: i) diagnoses were confirmed via histological testing; ii) patients did not exhibit distant metastasis; iii) patients had no severe complications or other malignancies. Staging of tumors was based on criteria stipulated by the Barcelona Clinic Liver Cancer (BCLC) staging system (22). Overall survival (OS) was defined as duration from the time of liver resection to that of death or the last follow-up. Progression-free survival (PFS) was defined as duration from the date of resection without any tumor progression, including deterioration of liver function, recurrence or metastasis. Baseline characteristics and survival data, based on follow-ups of all enrolled patients, were collected for statistical analyses. Resected tissues were prepared for studies aimed at the determination of protein expression and isolation of primary HCC cells.
Western blot analysis. Resected tissues were homogenized in RIPA lysis buffer (Beyotime) to obtain total protein, the concentration of which was measured using a BCA kit (Thermo Fisher Scientific, Inc.). An amount of $10 \mu \mathrm{g}$ of protein in each sample were adjusted to the same volume and separated via $10 \%$ SDS-PAGE. Proteins in the gel were electrically transferred onto a polyvinylidene difluoride (PVDF) membrane (Millipore). Unoccupied sites on the membrane were blocked using 5\% milk for $1 \mathrm{~h}$, following which the PVDF membrane was trimmed into pieces according to the relative molecular weights of the target proteins. Trimmed membranes were immersed in solutions containing primary antibodies (all provided by Abcam) for Beclin-1 (dilution 1:1,000, cat. no. ab210498), ASPP2 (dilution 1:2,000, cat. no. ab181377), LC3-I (dilution 1:800, cat. no. ab62721), LC3-II (dilution 1:1,000, cat. no. ab51520), cleaved caspase 3 (dilution 1:1,500, cat. no. ab32042), cleaved caspase 8 (dilution 1:2,000, cat. no. ab119809) and GAPDH (dilution 1:2,000, cat. no. ab181602) for $12 \mathrm{~h}$ at $4^{\circ} \mathrm{C}$. Next, immunoblots on the PVDF membrane were further probed by incubating with corresponding secondary antibodies (dilution 1:2,000, goat anti-rabbit IgG H\&L, cat. no. ab150077 and goat anti-mouse $\mathrm{IgG}$, cat. no. ab197767) for $1 \mathrm{~h}$ at room temperature, followed by measurement of the protein signals via the Odyssey System (Licor Biosciences, Germany) and band intensities using the BioRad Image Lab Software 3.0 (Bio-Rad Laboratories).

Cultivation of primary HCC cells and a liver cancer cell line. Liver cancer (HepG2) cells were cultivated in Dulbecco's modified Eagle's medium (DMEM) (Gibco; Thermo Fisher Scientific, Inc.) with $10 \%$ fetal bovine serum, FBS at $37^{\circ} \mathrm{C}$ under $5 \% \mathrm{CO}_{2}$.

Tumor as well as normal specimens were collected from the primary tumor and tumor-surrounding parenchyma in pre-cooled DMEM-F12 (10\% FBS) of 5 HCC patients (Table I). Next, the cells were dissociated from the tissues in pre-heated $0.1 \%$ collagenase IV, followed by filtration and resuspension of the cell pellet in cool medium. After 3 rounds of centrifugation at $81 \mathrm{x}$ g for $12 \mathrm{~min}$, the hepatocytes were separated as a pellet. The pellet containing liver cells was resuspended in ice-cold DMEM-F12 with 20\% FBS, following which triple centrifugation was performed at $81 \mathrm{xg}$ for $12 \mathrm{~min}$ at $4^{\circ} \mathrm{C}$ in order to separate the purified hepatocyte population (pellet) from the non-parenchymal cells (supernatant). The pellet contained partially purified hepatocytes. To further purify the hepatocytes, the pellet was placed in a dish without any collagen I. The supernatant was carefully removed $4 \mathrm{~h}$ later, and tumor cells and non-tumor cells were inoculated on a 24-well plate for $48 \mathrm{~h}$. At $16 \mathrm{~h}$ before co-culture, the original medium was refreshed, and cells were rinsed twice in PBS. Cell culture was continued in medium containing 1\% FBS. Hydroxychloroquine (HCQ) $(10 \mu \mathrm{M})$ was administrated to inhibit the autophagy process in in vivo experiment to explore the role of autophagy in the cellular protection in primary HCC cells or in HepG2 cells.

MTT assay. MTT assay was used to measure the cell viability as previously reported (14). Cells were seeded in 96-well plates $\left(2 \sim 3 \times 10^{3}\right.$ cells/well $)$, and 5 -FU $(20 \mu \mathrm{g} / \mathrm{ml})$ or HCQ $(10 \mu \mathrm{M})$ 
Table I. Characteristics of the 5 patients from whom primary HCC cells were isolated.

\begin{tabular}{|c|c|c|c|c|c|c|}
\hline No. of patients & Age (years) & Sex & BCLC staging & Child-Pugh classification & AFP (ng/ml) & PVTT (yes or no) \\
\hline 213 & 56 & Male & $\mathrm{B}$ & $\mathrm{B}$ & $1,385.50$ & No \\
\hline 267 & 61 & Male & $\mathrm{C}$ & $\mathrm{C}$ & $2,490.23$ & No \\
\hline 367 & 37 & Male & $\mathrm{C}$ & $\mathrm{C}$ & 980.89 & Yes \\
\hline 463 & 58 & Male & $\mathrm{C}$ & $\mathrm{B}$ & $2,321.23$ & No \\
\hline 554 & 69 & Male & $\mathrm{B}$ & $\mathrm{B}$ & $1,656.76$ & No \\
\hline
\end{tabular}

HCC, hepatocellular carcinoma; BCLC, Barcelona Clinic Liver Cancer; AFP, $\alpha$-fetoprotein; PVTT, portal vein tumor thrombus.

was added for 2-4 days. Hypoxic stimulation was given at a oxygen concentration of $5 \%\left(5 \% \mathrm{CO}_{2}\right.$ and $\left.90 \% \mathrm{~N}_{2}\right)$. The cells were cultured with $100 \mu \mathrm{l}$ sterile MTT dye (Sigma-Aldrich; Merck KGaA) for $4 \mathrm{~h}$, followed by the removal of medium and supplementation with $150 \mu \mathrm{l}$ of DMSO (dimethyl sulphoxide). After shaking, the absorbance was measured.

Immunohistochemical staining. The carcinoma tissues from patients were immediately fixed by $5 \%$ formalin and then embedded in paraffin blocks. Before immunohistochemical staining, antigen was retrieved according to thermal remediation method and the slides were incubated with the $3 \%$ goat serum for $30 \mathrm{~min}$. The paraffin block was cut into $5-\mu \mathrm{m}$ sections and then incubated with primary antibody for rat anti-human ASPP2 (BD Pharmingen) in $5 \%$ rabbit serum overnight at $4^{\circ} \mathrm{C}$ followed by washing and incubation with $\mathrm{ABC}$ (avidin-linked biotin complex) rabbit anti-rat Ig. The signals were detected using DAB (3,3'-diaminobenzidine) substrate and hematoxylin was used for counterstaining. The slides were dehydrated, coverslipped, and observed under a Zeiss microscope (Zeiss, Germany). A negative control in the absence of primary antibodies and incubated with isotype-matched immunoglobulins was also used for immunostaining. All captured images were analyzed by ImageJ softwared (National Institutes of Health, Bethesda, MD, USA) to quantify the expression level according the ratio of fluorescent intensity of primary antibody-stained section to negative control. The mean of expression level was used to divide the subjects into two groups.

Patient follow-up. Follow-up of patients ended in February 2018. During the follow-up, patients were required to attend an examination every 2 months to determine $\alpha$-fetoprotein (AFP) levels in the serum, submit to imaging examinations including abdominal ultrasonography and undergo CT or MRI scan every 6 months. In addition, an 18F-FDG PET-CT scan every 6 months was also suggested to clarify distant metastasis status.

Metastasis was evaluated via a combination of 18 F-FDG positron emission tomography and CT (PET-CT) every 6 months, following hepatectomy. Recurrence of HCC and liver complications were the major causes of death.

Statistical analysis. Data analysis was conducted using SPSS software 20.0 (IBM, Corp.). Relative expression of Beclin-1 and ASPP 2 are expressed as mean \pm standard deviation, and compared via the pairwise Student's t-test. Correlation between the Cezanne and staining scores of Beclin-1 and ASPP2 were detected using linear correlation analysis. Independent risk factors affecting 1-year survival of patients were identified via multiple logistics regression analysis, while survival was assessed via Kaplan-Meier curves. $\mathrm{P}<0.05$ was considered to indicate a statistically significant difference.

\section{Results}

Patient characteristics. In total, 165 patients who underwent direct surgical resection and 437 patients who underwent liver resection following TACE were retrospectively enrolled in the study. Among patients with direct resection, all patients were candidates for resection. Among the 437 patients who received TACE before resection, 205 patients were candidates for direct resection and 232 were unresectable at initiation. Baseline characteristics of the unresectable and resectable patients are presented (Tables II and III). Baseline characteristics of the unresectable HCC patients were compared before surgical resection, and no significant differences were found between the baseline characteristics of the high ASPP2 expression and low ASPP2 expression groups. The tested baseline characteristics included age, sex, platelet count (PLT), serum levels of blood urea nitrogen (BUN), tumor size, number of cancerous nodules, histological grading, Child-Pugh classification, microvessel invasion (MVI), portal vein tumor thrombus and ascites. However, AFP levels were significantly higher in unresectable HCC patients with a higher ASPP2 expression. Among the 437 patients who underwent surgical resection following TACE, 267 patients were considered as responders, defined as exhibiting a complete response (CR), or a partial response (PR) according to modified RECIST criteria for HCC (23). The remaining 170 patients exhibited stable disease (SD) or progressive disease (PD), defined according to modified RECIST criteria. In resectable and unresectable patients, a higher ASPP2 expression was associated with a positive therapeutic response to TACE according to modified RECIST criteria (Tables II and III).

Expression of ASPP2 and Beclin-1 in the HCC tissues of patients with or without TACE. In order to investigate ASPP2 expression in carcinoma tissues following TACE, carcinoma and para-carcinoma tissues were collected from patients who underwent surgical resection directly or following TACE. Next, ASPP2 expression in carcinoma and para-carcinoma tissues was assessed by western blot 
Table II. Baseline characteristics of the unresectable HCC patients with high or low expression of ASPP2 (n=232).

\begin{tabular}{|c|c|c|c|}
\hline Variable & High ASPP2 expression $(n=106)$ & Low ASPP2 expression $(n=126)$ & P-value \\
\hline Mean age, years & $54.61 \pm 10.54$ & $56.85 \pm 12.67$ & NS \\
\hline Gender, male n $(\%)$ & 77 (72.64) & $80(63.49)$ & NS \\
\hline $\operatorname{PLT}\left(10^{3} / \mu 1\right)$ & $187.34 \pm 68.91$ & $179.14 \pm 76.16$ & NS \\
\hline BUN (mg/dl) & $18.78 \pm 8.18$ & $17.67 \pm 10.15$ & NS \\
\hline Child-Pugh classification, n (\%) & & & NS \\
\hline A & $28(26.40)$ & $45(35.71)$ & \\
\hline $\mathrm{B}$ & $66(62.26)$ & $60(47.61)$ & \\
\hline $\mathrm{C}$ & $12(11.34)$ & $21(16.68)$ & \\
\hline Hepatitis B, n (\%) & $80(75.47)$ & $88(69.84)$ & \\
\hline $\operatorname{AFP}(\mathrm{ng} / \mathrm{ml})$ & $2,376.98 \pm 317.12$ & $2,087.33 \pm 286.89$ & $0.036^{\mathrm{a}}$ \\
\hline Tumor size, n (\%) & & & NS \\
\hline$\leq 5 \mathrm{~cm}$ & $59(55.66)$ & $54(42.86)$ & \\
\hline$>5 \mathrm{~cm}$ & $47(44.34)$ & $72(57.14)$ & \\
\hline BCLC stage, n (\%) & & & NS \\
\hline $0-\mathrm{B}$ & $63(59.33)$ & $57(45.24)$ & \\
\hline C-D & $43(40.67)$ & $65(54.76)$ & \\
\hline Histological grading, $\mathrm{n}(\%)$ & & & NS \\
\hline Well & $25(23.54)$ & $36(28.57)$ & \\
\hline Moderate & $48(45.67)$ & $51(40.48)$ & \\
\hline Poor & $33(30.79)$ & $38(30.16)$ & \\
\hline MVI \% & $48(45.67)$ & $66(52.38)$ & NS \\
\hline Portal vein thrombi, n (\%) & $28(26.41)$ & $47(37.30)$ & NS \\
\hline Ascites $\mathrm{n}, \%$ & $44(41.51)$ & $50(39.68)$ & NS \\
\hline Objective therapeutic response, $\mathrm{n}(\%)$ & & & $0.017^{\mathrm{a}}$ \\
\hline $\mathrm{CR}$ and $\mathrm{PR}$ & $62(58.49)$ & $26(20.63)$ & \\
\hline $\mathrm{SD}$ and $\mathrm{PD}$ & $44(41.51)$ & $100(79.37)$ & \\
\hline
\end{tabular}

Chi-square test or t-test were used for statistical analysis. ${ }^{a} \mathrm{P}<0.05$ indicates statistical significance. HCC, hepatocellular carcinoma; ASPP2, apoptosis-stimulating p53 protein 2; PLT, platelet level; BUN, blood urea nitrogen; AFP, $\alpha$-fetoprotein; BCLC, Barcelona Clinic Liver Cancer; MVI, microvessel invasion; CR, complete response; PR, partial response; SD, stable disease; PD, progressive disease; NS, not significant.

assay. ASPP2 expression in carcinoma tissues of patients who received surgical resection either directly or following TACE was significantly lower, compared with that in the para-carcinoma tissues (Fig. 1A and B). The ratio of ASPP2 in para-carcinoma to carcinoma tissues (Para-CA/CA) was significantly higher in patients receiving surgical resection following TACE, than that in those receiving surgical resections directly (Fig. 1C). Beclin-1 expression in carcinoma tissues and para-carcinoma tissues of HCC patients who received surgical resection directly or following TACE was assessed by western blot assay. Beclin-1 expression in carcinoma tissues was observably increased, compared with that of para-carcinoma tissues in HCC patients following TACE. However, there was no observable difference in Beclin-1 expression between carcinoma and para-carcinoma tissues from HCC patients who did not undergo TACE before surgical resection (Fig. 1D and E). In addition, Beclin-1 expression in the histological samples of patients who underwent TACE, was negatively correlated with ASPP2 expression (Fig. 1F). These data indirectly demonstrated that a reduction in ASPP-2 expression, which occurred in the carcinoma tissues of patients following TACE, was associated with autophagy which was dependent on Beclin-1.

Culture in hypoxia and chemotherapy-induced cell apoptosis promote autophagy in HepG2 liver cancer cells and primary HCC cells from HCC patients. Expression of LC3-II and LC3-I in carcinoma and para-carcinoma tissues of samples from patients who underwent surgical resection following TACE was assessed by western blot assay. Results indicated that LC3-II expression was increased (Fig. 2A) and the LC3-II to LC3-I ratio was higher in carcinoma tissues compared with those in para-carcinoma tissues (Fig. 2B), suggesting that autophagy flux in cancer cells was increased following TACE. Examination of primary HCC cells isolated from a patient who underwent palliative surgical resection following TACE failure indicated that administration of $5-\mathrm{FU}$ at a low concentration $(20 \mu \mathrm{g} / \mathrm{ml})$ and in vitro hypoxia stimulation led to an increase in LC3-II expression and a higher ratio of LC3-II/LC3-I (Fig. 2C). Application of the autophagy inhibitor, hydroxychloroquine 
Table III. Baseline characteristics of the resectable HCC patients with high or low expression of ASPP2 (n=205).

\begin{tabular}{|c|c|c|c|}
\hline Variable & High ASPP2 expression $(n=93)$ & Low ASPP2 expression $(n=112)$ & P-value \\
\hline Mean age, years & $54.23 \pm 13.64$ & $53.85 \pm 10.61$ & NS \\
\hline Gender, male n (\%) & $59(63.45)$ & $77(68.91)$ & NS \\
\hline $\operatorname{PLT}\left(10^{3} / \mu 1\right)$ & $194.34 \pm 46.81$ & $197.14 \pm 51.56$ & NS \\
\hline BUN (mg/dl) & $15.61 \pm 7.18$ & $16.71 \pm 9.62$ & NS \\
\hline Child-Pugh classification, n (\%) & & & NS \\
\hline A & $35(37.45)$ & $47(42.34)$ & \\
\hline $\mathrm{B}$ & $52(56.12)$ & $50(44.51)$ & \\
\hline $\mathrm{C}$ & $6(5.43)$ & $15(13.15)$ & \\
\hline Hepatitis B, n (\%) & $76(82.13)$ & 74.78 & \\
\hline $\operatorname{AFP}(\mathrm{ng} / \mathrm{ml})$ & $1,965.98 \pm 567.12$ & $1,876.33 \pm 789.10$ & NS \\
\hline Tumor size, n (\%) & & & NS \\
\hline$\leq 5 \mathrm{~cm}$ & $32(34.12)$ & $30(26.61)$ & \\
\hline$>5 \mathrm{~cm}$ & $61(65.88)$ & $82(73.21)$ & \\
\hline BCLC stage, n (\%) & & & NS \\
\hline $0-\mathrm{B}$ & $81(87.12)$ & $93(83.17)$ & \\
\hline C-D & $12(12.88)$ & $19(16.83)$ & \\
\hline Histological grading, $\mathrm{n}(\%)$ & & & NS \\
\hline Well & $51(55.54)$ & $54(48.13)$ & \\
\hline Moderate & $24(25.67)$ & $36(32.47)$ & \\
\hline Poor & $18(18.89)$ & $32(20.40)$ & \\
\hline MVI, n $(\%)$ & $39(41.87)$ & $51(45.38)$ & NS \\
\hline Portal vein thrombi, n (\%) & $0(0.00)$ & $0(0.00)$ & NS \\
\hline Ascites, $\mathrm{n}$ & 12.67 & 16.83 & NS \\
\hline Objective therapeutic response, n (\%) & & & $0.024^{\mathrm{a}}$ \\
\hline $\mathrm{CR}$ and $\mathrm{PR}$ & $49(52.69)$ & $21(18.75)$ & \\
\hline SD and PD & $44(47.31)$ & $91(81.25)$ & \\
\hline
\end{tabular}

Chi-square test or t-test were used for statistical analysis. ${ }^{a} \mathrm{P}<0.05$, indicates statistical significance. HCC, hepatocellular carcinoma; ASPP2, apoptosis-stimulating p53 protein 2; PLT, platelet level; BUN, blood urea nitrogen; AFP, $\alpha$-fetoprotein; BCLC, Barcelona Clinic Liver Cancer; MVI, microvessel invasion; CR, complete response; PR, partial response; SD, stable disease; PD, progressive disease; NS, not significant.

(HCQ) $(10 \mu \mathrm{M})$, promoted apoptosis of primary HCC cells under hypoxic cultivation and 5-FU treatment, as evidenced by the increased expression of cleaved caspase-3 and cleaved caspase-8 (Fig. 2D) and inhibition of proliferation, as measured via MTT assays (Fig. 2E). Furthermore, we assessed the expression of LC3-II as well as the ratio of LC-3II to LC-3I in the HepG2 cell line, under 5-FU treatment and hypoxia cultivation. The results indicated a significant increase in the expression of LC3-II and a higher ratio of LC3-II to LC3-I which was markedly similar to that observed in the primary HCC cells (Fig. 2F). The application of the autophagy inhibitor did not result in the change of biological behavior, including apoptosis, proliferation, and chemotherapy resistance (data not shown). The above findings illustrated that autophagy initiated by hypoxia or a chemotherapy drug may promote the survival of primary $\mathrm{HCC}$ cells and protect them from adverse conditions.

Expression of ASPP2 predicts the prognosis of neoadjuvant $T A C E$ and liver resection. In order to verify the prognostic significance of ASPP2 expression in predicting the survival of HCC patients following TACE and surgical resection, we constructed a cohort in the 437 patients treated with TACE following surgical resection. We collected and analyzed data related to survival and objective response. Immunohistochemical (IHC) assays and histopathological examinations were used to obtain a semi-quantitative evaluation of ASPP2 expression in tissues. These patients were divided into a low expression group and a high expression group according to a cut-off value determined by median expression levels. As shown in Table IV, patients with a higher ASPP2 expression had a low 1-year mortality rate following combination therapy with surgical resection (high expression vs. low expression, 65.3 vs. $\left.26.7 \% ; \chi^{2}=12.91 ; \mathrm{P}=0.003\right)$. Multiple logistics regression revealed that low ASPP2 expression levels, MVI and histopathological grading of carcinoma differentiation were an independent risk factor for TACE failure. PVTT $(\mathrm{OR}=2.17 ; 95 \%$ CI 1.34-3.61; $\mathrm{P}=0.020)$, Child-Pugh classification $(\mathrm{OR}=2.76 ; 95 \% \mathrm{CI} 1.35-4.12 ; \mathrm{P}=0.031)$ and $\mathrm{ASPP} 2$ $(\mathrm{OR}=2.12 ; 95 \%$ CI 1.68-3.75; $\mathrm{P}=0.004)$ were independent 
A

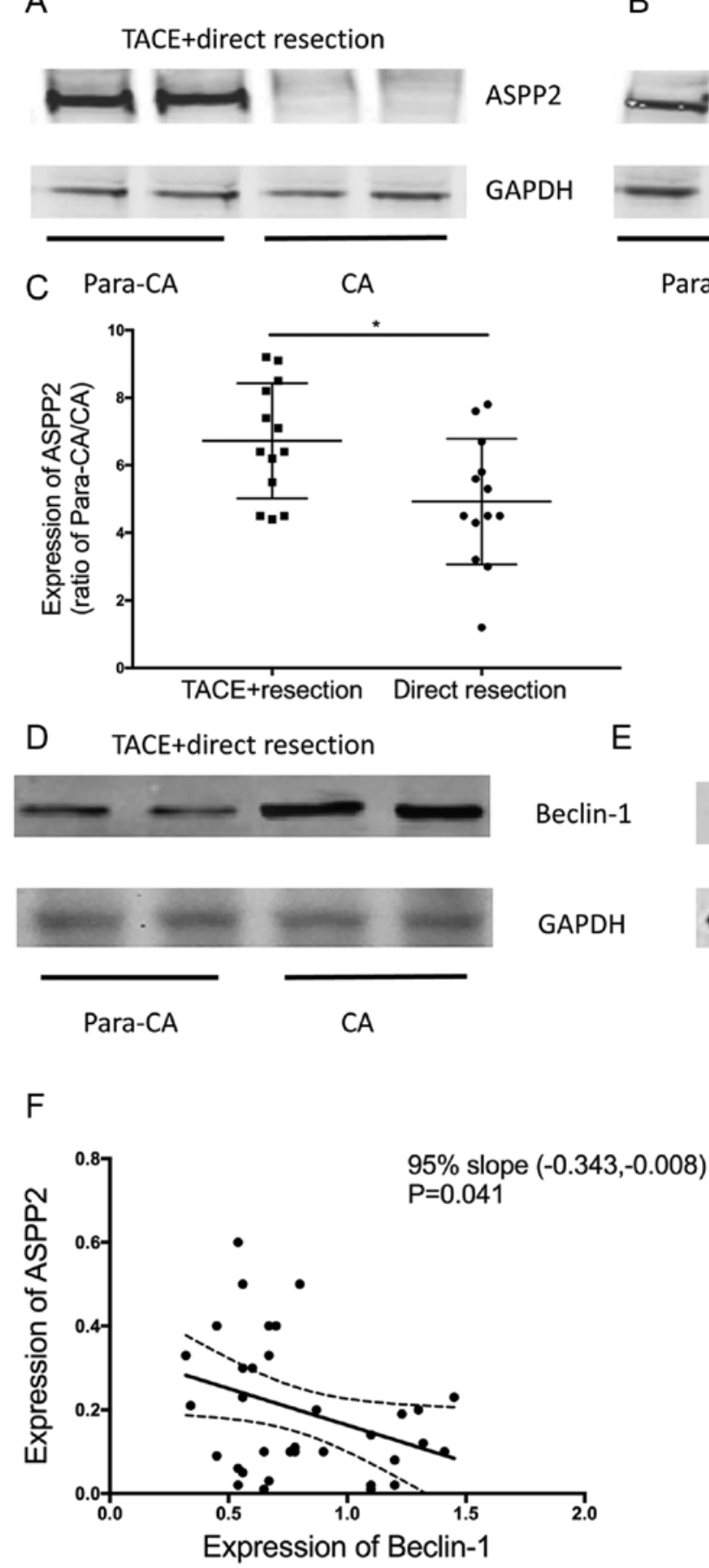

Direct resection
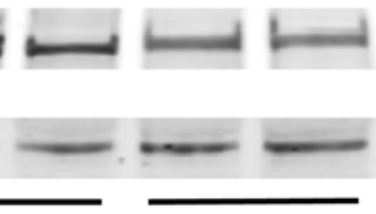

CA

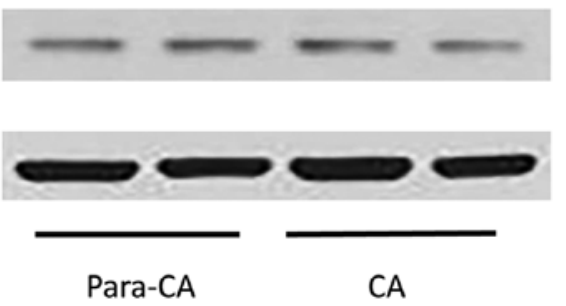

Direct resection 
A

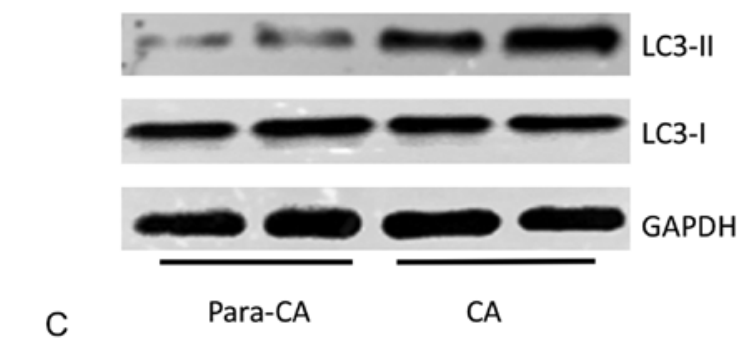

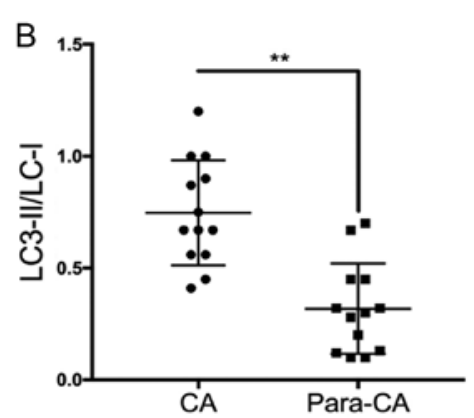

5-FU treatment
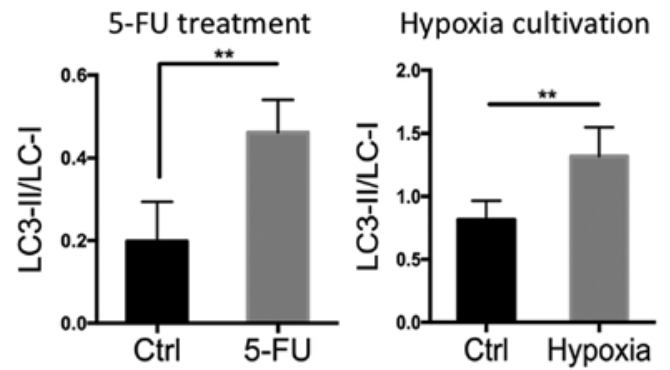

D

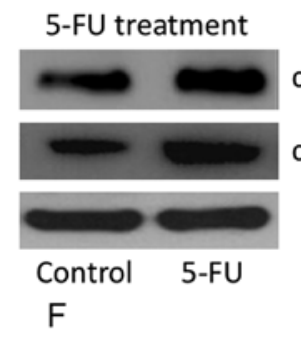

Primary HCC cells E

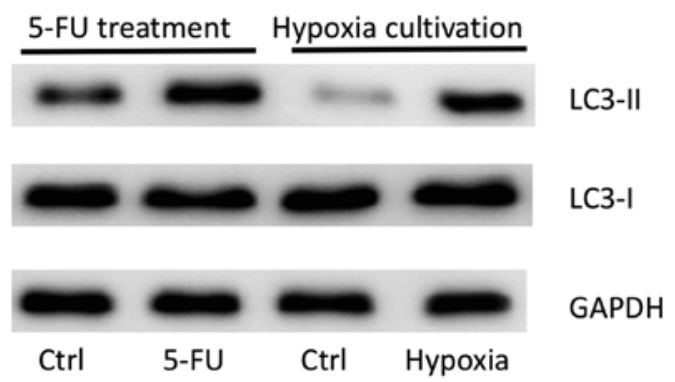

Hypoxia cultivation

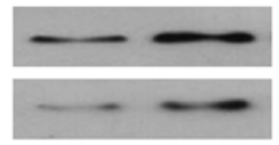

GAPDH

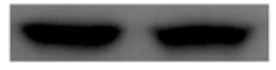

Control HCQ
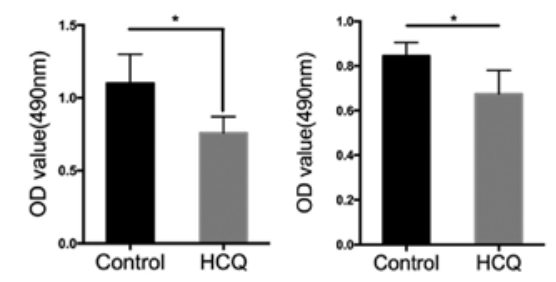

\section{5-FU treatment}

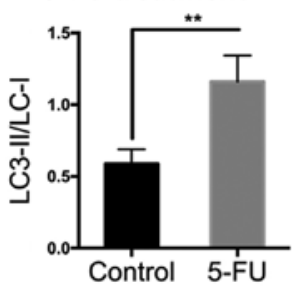

Hypoxia cultivation

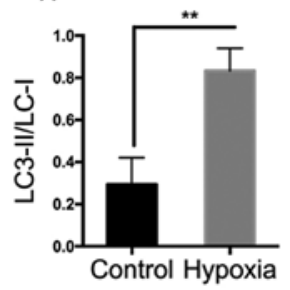

Figure 2. Autophagy-induced anti-apoptotic effect in primary HCC cells in vitro following hypoxia cultivation or administration of chemotherapy. (A) Expression of LC3-II and (B) ratio of LC3-II to LC3-I in para-carcinoma and carcinoma tissues of HCC patients following TACE. $n=5$, * $\mathrm{P}<0.01$. (C) 5 -FU treatment or hypoxia stimulation activated autophagy in the HCC primary cells of patients without an objective therapeutic response following TACE. n=5, ${ }^{* *} \mathrm{P}<0.01$. (D) Inhibitor of autophagy, HCQ (hydroxychloroquine), promoted the expression of cleaved caspase-3 and cleaved caspase-8 in the HCC primary cells of patients without an objective therapeutic response following TACE measured by western blot analysis. n=5. (E) Inhibitor of autophagy, HCQ (hydroxychloroquine), repressed proliferation of HCC primary cells of patients without objective therapeutic response following TACE measured by MTT assay. $\mathrm{n}=5$, ${ }^{*} \mathrm{P}<0.05$. (F) 5-FU treatment or hypoxia stimulation activated autophagy in the HepG2 cell line. ${ }^{* *} \mathrm{P}<0.01$. All experiments were repeated at least thrice. Student's t-test was used for statistical analysis. $\mathrm{P}<0.05$ indicates statistical difference. TACE, transcatheter arterial chemoembolization; HCC, hepatocellular carcinoma.

therapy followed by liver resection, regardless of the anatomic condition at initiation.

\section{Discussion}

Transcatheter arterial chemoembolization (TACE) is considered as a first-line therapeutic strategy for intermediate stage hepatocellular carcinoma (HCC) in the EASL-EORTC clinical practice guideline (24). For HCC patients considered unresectable at initiation, TACE is performed to downstage HCC prior to surgical resection $(1,2)$. Whether TACE is indicated for resectable HCC patient, as well as whether such patients would benefit from TACE remains a controversial topic. A meta-analysis based on HCC patients with macrovascular invasion reported that administration of TACE was correlated with a lower 1-year and 3-year survival rate compared to resection (25). These data demonstrated that refractoriness of TACE may have an adverse influence on 
Table IV. Multiple logistics regression analysis for the independent risk factor of 1-year mortality rate.

\begin{tabular}{|c|c|c|c|}
\hline Variable & P-value & OR & $95 \% \mathrm{CI}$ \\
\hline PVTT (no vs. yes) & $0.020^{\mathrm{a}}$ & 2.17 & $1.34-3.61$ \\
\hline Expression of ASPP2 (high vs. low expression) & $0.004^{\mathrm{a}}$ & 2.12 & $1.68-3.75$ \\
\hline Child-Pugh classification (classification $A=1$, classification $B=2$, classification $C=3$ ) & $0.031^{\mathrm{a}}$ & 2.76 & $1.68-3.75$ \\
\hline Tumor size $(\leq 5$ vs. $>5 \mathrm{~cm})$ & 0.078 & 1.54 & $0.87-1.98$ \\
\hline AFP levels $(\leq 1,500$ vs. $>1,500 \mathrm{ng} / \mathrm{ml})$ & 0.31 & 1.21 & $0.76-1.88$ \\
\hline Age $(\leq 50$ vs. $>50$ years $)$ & 0.21 & 1.23 & $0.65-1.78$ \\
\hline BCLC stage (0-B vs. C-D) & 0.067 & 1.98 & $0.91-2.80$ \\
\hline MVI (no vs. yes) & 0.058 & 1.58 & $0.96-2.77$ \\
\hline
\end{tabular}

Multiple logistics regression analysis was used for statistical analysis and ${ }^{\mathrm{P}}<0.05$, indicates statistical significance. OR, odds ratio; CI, confidence interval; PVTT, portal vein tumor thrombus; ASPP2, apoptosis-stimulating p53 protein 2; AFP, $\alpha$-fetoprotein; BCLC, Barcelona Clinic Liver Cancer; MVI, microvessel invasion.

prognosis and that TACE should be administrated only to those patients conforming to selective criteria. For recurrent but resectable $\mathrm{HCC}$, resection with preoperative TACE may not improve prognosis compared to single resection. It has been confirmed that TACE may improve the prognosis for unresectable patients compared to supportive therapy, and may lead to the downstaging of HCC in some patients who are considered unresectable at initiation (8). In those patients for whom HCC was not down-staged following TACE, disease-free survival was significantly worse than that of patients with a remarkable response to TACE (8).

Furthermore, in spite of the known efficacy and a level of safety associated with it, administration of TACE in intermediate HCC patients is beset with several concerns and limitations $(1,26,27)$. The most important of these is an incomplete and weak response to TACE due to insufficient arterial supply or large tumor size $(>5 \mathrm{~cm})(26)$. The second issue is related to a hypoxic microenvironment, which inevitably results from arterial embolization, that promotes angiogenic factors such as the vascular endothelial growth factor (VEGF) $(26,27)$. The surge in serum VEGF levels following TACE was found to be correlated with tumor size, vascular invasion and patient survival. Tolerance of hypo-perfusion of chemotherapy drugs in a hypoxia environment in HCC patients with larger tumors may increase their malignant biological behavior and induce therapeutic resistance to TACE. It has been reported that the expression of hypoxia-induced factor 1 (HIF-1) and VEGF was increased following TACE $(28,29)$. Increased expression of some stemness markers was also found in HCC tissues following TACE. These compensatory changes in biological behavior may reduce sensitivity to TACE and aggravate resistance to regional chemotherapy. In addition, TACE may eventually result in the deterioration of liver function, damage to the hepatic artery, an increased post-embolization liver failure risk and, most importantly, a poor quality of life, leading to a poor prognosis in those patients who do not respond to TACE and do not exhibit tumor downstaging (30). As shown in many previous investigations, the clinical benefits are correlated with the presence of objective response and are evident only in patients with Child-Pugh B, European Cooperative Oncology
Group (ECOG) status 1, who did not present with severe liver cirrhosis (31). Therefore, whether patients benefit from liver resection following preoperative TACE may be dependent on fundamental liver function and inhibition of compensatory mechanisms underlying resistance to chemotherapy and ischemia.

In solid tumors, autophagy functions as a survival mechanism against various stressors including metabolic stress, starvation, hypoxia, chemotherapy, and radiotherapy (12-15). In physiological situations, autophagy plays an important role in organelle turnover, protein degradation, cellular differentiation and aging. Under stress, autophagy protects cells by eliminating damaged organelles and proteins via autophagosomes. Under mild and physiological hypoxia $\left(0.19-3 \% \mathrm{O}_{2}\right)$, autophagic response is dependent on HIF-1, which has an anti-apoptotic effect on cancer cells $(32,33)$. Autophagy may promote cell survival. In HCC, under nutrition derivation Beclin1-induced autophagy, chemotherapy resistance in pancreatic cancer cells was increased as well (34). Moreover, Beclin-1-induced autophagy mediated cell survival in leukemia cells (35). Previous research indicated that activation of autophagy was correlated with cell survival as well as resistance to adverse conditions in cancer cells (36). In addition, according to a previous study, Beclin-1, considered as a molecular autophagy marker, may be downregulated by apoptosis-stimulating p53 protein 2 (ASPP2), which was considered as a transcriptional cofactor of p53 and can act as a tumor suppressor. Therefore, downregulation of ASPP may protect cancer cells from autophagy instead of conventional p53-related signaling $(16,17)$. Chen et al (37) found that mechanically, ASPP2 binds to BECN1, leading to a decrease in binding of PIK $3 \mathrm{C} 3$ and the UV radiation resistance-associated gene (UVRAG), and an increase in the binding of Rubicon in the PIK3C3 complex. The present study demonstrated that downregulation of ASPP2 enhanced pro-survival, chemoresistant properties of HCC cells via autophagy, both in vitro and in vivo. In HCC, administration of TACE led to local ischemia of carcinoma tissues and the regional release of chemotherapy. Therefore, we speculated that autophagy activation in carcinoma may promote resistance to chemotherapy and hypoxia environments following TACE, leading to an incomplete 
A

Progression-free survival

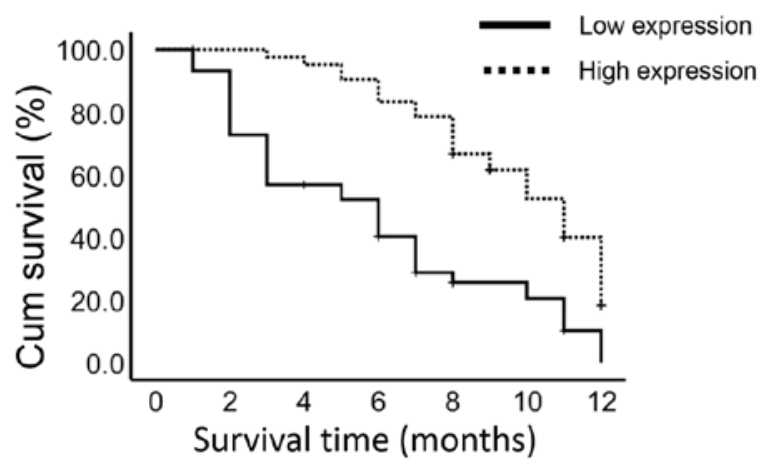

B

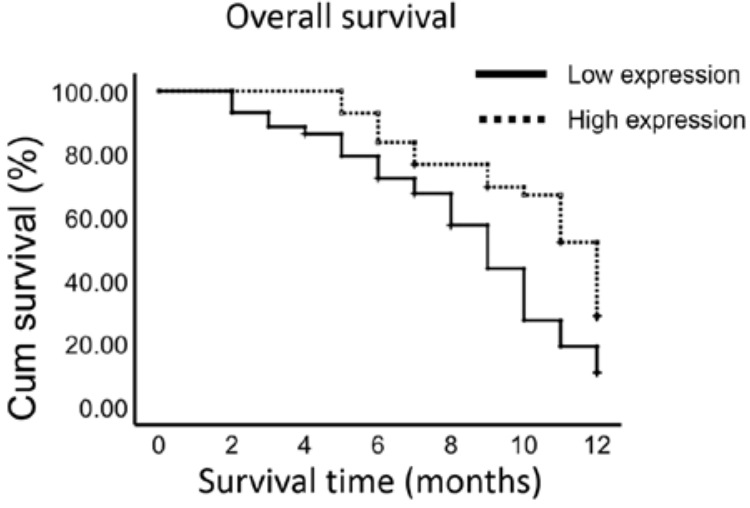

C

Progression-free survival
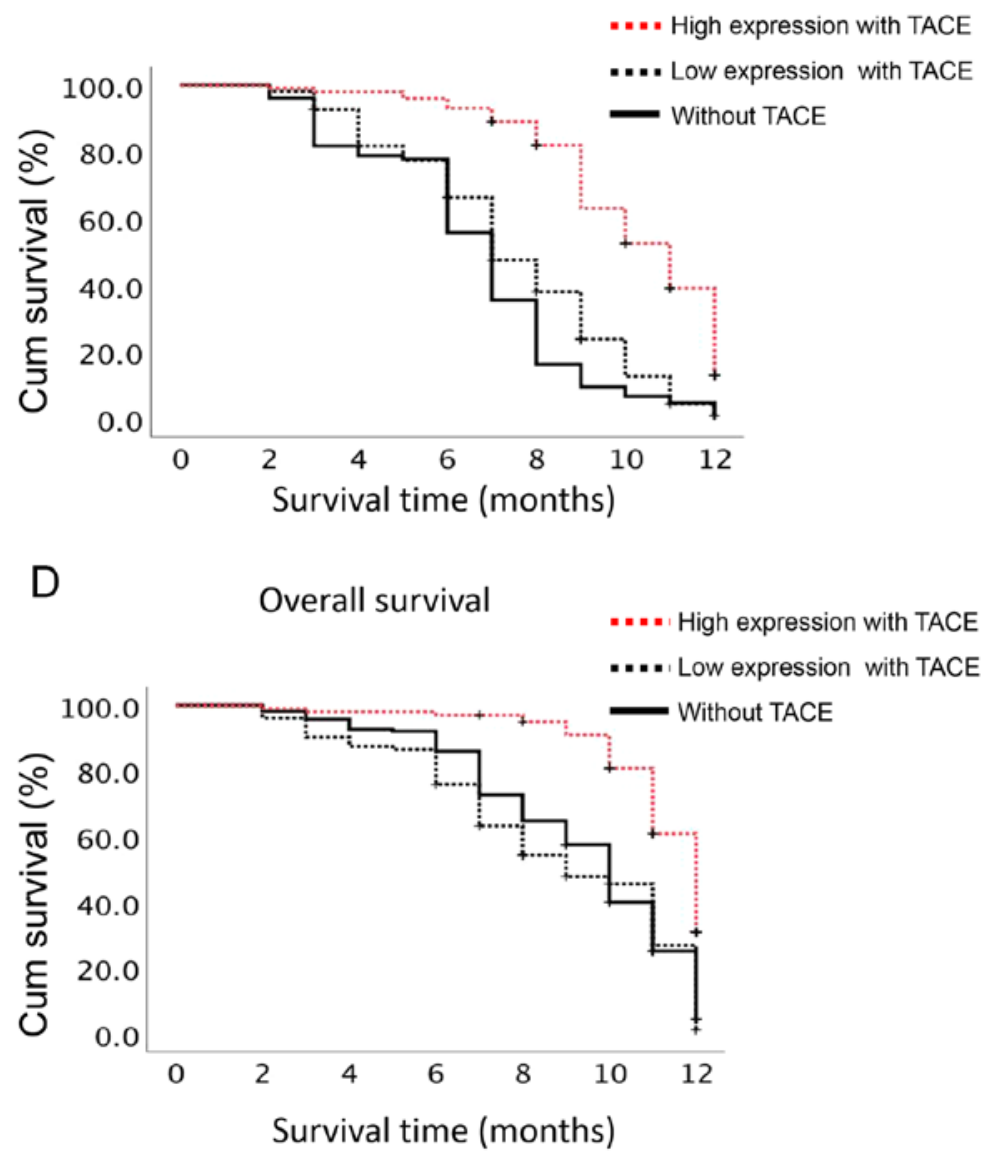

Figure 3. Survival analysis and expression of ASPP2 in the HCC patients after TACE. (A) The 1-year progression-free survival in unresectable HCC patients at initiation after neoadjuvant TACE combination and resection in all subjects with high expression (dotted line) and low expression of ASPP2 (solid line). (B) The 1-year overall survival in unresectable HCC patients at initiation following neoadjuvant TACE combination and resection in all subjects with high expression (dotted line) and low expression of ASPP2 (solid line). (C) The 1-year progression-free survival in resectable HCC patients following neoadjuvant TACE combination and resection in three groups subjects, including patients who were administered TACE with high expression (red dotted line) and low expression (black dotted line) of ASPP2 or who did not received neoadjunct TACE (black solid line). (D) The 1-year over survival in resectable HCC patients following neoadjuvant TACE combination and resection in three groups subjects, including patients who were administered TACE with high expression and low expression of ASPP2 or not. Survival analysis was used for the statistical analysis. The survival curve is depicted according to the Kaplan-Meier method. Red dotted line, patients administered TACE and with a high expression of ASPP2; Black dotted line, patients administered TACE and with a low expression of ASPP2; Black solid line, patients without TACE administration. ASPP2, apoptosis-stimulating p53 protein 2; TACE, transcatheter arterial chemoembolization; HCC, hepatocellular carcinoma.

response to TACE. We found that inhibition of autophagy in primary HCC cells of a patient exhibiting TACE failure in vitro, promoted apoptosis and attenuated chemotherapy resistance. Our results demonstrated that activation of autophagy in HCC cells under stress, protected cancer cells from apoptosis. It was also observed that decreased ASPP2 expression in carcinoma tissues of HCC samples in patients following TACE, was associated with increased Beclin-1 expression. 
These findings derived from basic research suggest that ASPP2 expression may act as a predictor of prognosis following TACE. We further measured ASPP2 expression in HCC tissues from HCC patients following TACE, where a high ASPP2 expression was associated with a higher objective therapeutic response rate, indicating a better prognosis for unresectable HCC patients. Furthermore, administration of neoadjuvant TACE improved survival only in resectable patients with a high ASPP2 expression. Clinical observation data combined with the above stated findings from basic experiments demonstrated that patients with a high ASPP2 expression following TACE show a higher response rate than those with a low expression, which may be associated with increased sensitivity to TACE after inhibition of autophagy via downstream tumor-suppressor p53 or ASPP2/Beclin1 signaling pathways.

In conclusion, our study provides a novel biomarker for predicting prognosis and proposes a potential approach for choosing suitable patients who may benefit from TACE combined with resection.

\section{Acknowledgements}

Not applicable.

\section{Funding}

No funding was received.

\section{Availability of data and materials}

The datasets used and/or analyzed during the current study are available from the corresponding author on reasonable request.

\section{Authors' contributions}

JM and ZT conceived the study and designed the experiments. XP and FM contributed to the data collection; performed the data analysis and interpreted the results. JM wrote the manuscript; JM and ZT contributed to the critical revision of the article. All authors read and approved the final manuscript and agree to be accountable for all aspects of the research in ensuring that the accuracy or integrity of any part of the work are appropriately investigated and resolved.

\section{Ethics approval and consent to participate}

This observational study was approved by the Medical Ethics Committee of The People's Hospital of Danyang (Danyang, Jiangsu). Written consent for collection of tissue samples was acquired from all patients prior to surgery.

\section{Patient consent for publication}

Not applicable.

\section{Competing interests}

The authors declare that they have no competing interests.

\section{References}

1. Galle PR, Tovoli F, Foerster F, Wörns MA, Cucchetti A and Bolondi L: The treatment of intermediate stage tumours beyond TACE: From surgery to systemic therapy. J Hepatol 67: 173-183, 2017.

2. Katsanos K, Kitrou P, Spiliopoulos S, Maroulis I, Petsas T and Karnabatidis D: Comparative effectiveness of different transarterial embolization therapies alone or in combination with local ablative or adjuvant systemic treatments for unresectable hepatocellular carcinoma: A network meta-analysis of randomized controlled trials. PLoS One 12: e0184597, 2017.

3. Ye JZ, Chen JZ, Li ZH, Bai T, Chen J, Zhu SL, Li LQ and Wu FX: Efficacy of postoperative adjuvant transcatheter arterial chemoembolization in hepatocellular carcinoma patients with microvascular invasion. World J Gastroenterol 23: 7415-7424, 2017.

4. Jianyong L, Jinjing Z, Lunan Y, Jingqiang Z, Wentao W, Yong Z, Bo L, Tianfu W and Jiaying Y: Preoperative adjuvant transarterial chemoembolization cannot improve the long term outcome of radical therapies for hepatocellular carcinoma. Sci Rep 7: 41624, 2017.

5. Ha TY, Hwang S, Lee YJ, Kim KH, Ko GY, Ii Gwon D, Ahn CS, Moon DB, Song GW, Jung DH, et al: Absence of benefit of transcatheter arterial chemoembolization (TACE) in patients with resectable solitary hepatocellular carcinoma. World J Surg 40: 1200-1210, 2016.

6. Wang W, Bai W, Wang E, Zhao Y, Liu L, Yang M, Cai H, Xia D, Zhang L, Niu J, et al: mRECIST response combined with sorafenib-related adverse events is superior to either criterion alone in predicting survival in HCC patients treated with TACE plus sorafenib. Int J Cancer 140: 390-399, 2017.

7. Shi HY, Wang SN, Wang SC, Chuang SC, Chen CM and Lee KT: Preoperative transarterial chemoembolization and resection for hepatocellular carcinoma: A nationwide Taiwan database analysis of long-term outcome predictors. J Surg Oncol 109: 487-493, 2014.

8. Majno PE, Adam R, Bismuth H, Castaing D, Ariche A, Krissat J, Perrin H and Azoulay D: Influence of preoperative transarterial lipiodol chemoembolization on resection and transplantation for hepatocellular carcinoma in patients with cirrhosis. Ann Surg 226: 688-703, 1997.

9. Liu K, Min XL, Peng J, Yang K, Yang L and Zhang XM: The changes of HIF- $1 \alpha$ and VEGF expression after TACE in patients with hepatocellular carcinoma. J Clin Med Res 8: 297-302, 2016.

10. Yuan G, Zeng CL, Zhu DD and Shi XJ: Influences of RFA combined with TACE on the HIF-1 $\alpha$ and EGR level of patients with primary hepatic carcinoma. Eur Rev Med Pharmacol Sci 21: 1738-1745, 2017.

11. Xu W, Kwon JH, Moon YH, Kim YB, Yu YS, Lee N, Choi KY, Kim YS, Park YK, Kim BW and Wang HJ: Influence of preoperative transcatheter arterial chemoembolization on gene expression in the HIF-1 $\alpha$ pathway in patients with hepatocellular carcinoma. J Cancer Res Clin Oncol 140: 1507-1515, 2014.

12. Pagotto A, Pilotto G, Mazzoldi EL, Nicoletto MO, Frezzini S, Pastò A and Amadori A: Autophagy inhibition reduces chemoresistance and tumorigenic potential of human ovarian cancer stem cells. Cell Death Dis 8: e2943, 2017.

13. Jawhari S, Ratinaud MH and Verdier M: Glioblastoma, hypoxia and autophagy: A survival-prone 'ménage-à-trois'. Cell Death Dis 7: e2434, 2016.

14. Zhou C, Ma K, Gao R, Mu C, Chen L, Liu Q, Luo Q, Feng D, Zhu Y and Chen Q: Regulation of mATG9 trafficking by Src- and ULK1-mediated phosphorylation in basal and starvation-induced autophagy. Cell Res 27: 184-201, 2017.

15. White E, Mehnert JM and Chan CS: Autophagy, metabolism, and cancer. Clin Cancer Res 21: 5037-5046, 2015.

16. Vives V, Slee EA and Lu X: ASPP2: A gene that controls life and death in vivo. Cell Cycle 5: 2187-2190, 2006.

17. Sullivan A and Lu X: ASPP: A new family of oncogenes and tumour suppressor genes. Br J Cancer 96: 196-200, 2007.

18. Yang T, Gao Y, Liu D, Wang Y, Wu J, Liu X, Shi Y and Chen D: ASPP2 enhances chemotherapeutic sensitivity through the down-regulation of XIAP expression in a p53 independent manner in hepatocellular carcinoma. Biochem Biophys Res Commun 508: 769-774, 2019.

19. Wu X, Feng X, Zhao X, Ma F, Liu N, Guo H, Li C, Du H and Zhang B: Role of Beclin-1-mediated autophagy in the survival of pediatric leukemia cells. Cell Physiol Biochem 39: 1827-1836, 2016. 
20. Ueno T, Saji S, Sugimoto M, Masuda N, Kuroi K, Sato N, Takei H, Yamamoto Y, Ohno S, Yamashita H, et al: Clinical significance of the expression of autophagy-associated marker, beclin 1, in breast cancer patients who received neoadjuvant endocrine therapy. BMC Cancer 16: 230, 2016.

21. Amaral C, Borges M, Melo S, da Silva ET, Correia-da-Silva G and Teixeira N: Apoptosis and autophagy in breast cancer cells following exemestane treatment. PLoS One 7: e42398, 2012.

22. Han K and Kim JH: Transarterial chemoembolization in hepatocellular carcinoma treatment: Barcelona clinic liver cancer staging system. World J Gastroenterol 21: 10327-10335, 2015.

23. Seymour L, Bogaerts J, Perrone A, Ford R, Schwartz LH Mandrekar S, Lin NU, Litière S, Dancey $\mathrm{J}$, Chen A, et al: iRECIST: Guidelines for response criteria for use in trials testing immunotherapeutics. Lancet Oncol 18: e143-e152, 2017.

24. European Association for the Study of the Liver; European Organisation for Research and Treatment of Cancer EASL-EORTC clinical practice guidelines: Management of hepatocellular carcinoma. J Hepatol 56: 908-943, 2012.

25. Chen J, Huang J, Chen M, Yang K, Chen J, Wang J, Xu L, Zhou Z and Zhang Y: Transcatheter arterial chemoembolization (TACE) versus hepatectomy in hepatocellular carcinoma with macrovascular invasion: A meta-analysis of 1,683 patients. J Cancer 8 : 2984-2991, 2017.

26. Sacco R, Tapete G, Simonetti N, Sellitri R, Natali V, Melissari S, Cabibbo G, Biscaglia L, Bresci G and Giacomelli L: Transarterial chemoembolization for the treatment of hepatocellular carcinoma: A review. J Hepatocell Carcinoma 4: 105-110, 2017.

27. Kudo M, Matsui O, Izumi N, Kadoya M, Okusaka T, Miyayama S, Yamakado K, Tsuchiya K, Ueshima K. Hiraoka A, et al: Transarterial chemoembolization failure/refractoriness: JSH-LCSGJ criteria 2014 update. Oncology 87 (Suppl 1): S22-S31, 2014

28. Li Z, Xue TQ and Chen XY: Predictive values of serum VEGF and CRP levels combined with contrast enhanced MRI in hepatocellular carcinoma patients after TACE. Am J Cancer Res 6 : 2375-2385, 2016

29. Guo LY, Zhu P and Jin XP: Association between the expression of HIF- $1 \alpha$ and VEGF and prognostic implications in primary liver cancer. Genet Mol Res 15: 15, 2016.
30. Waked I, Berhane S, Toyoda H, Chan SL, Stern N, Palmer D, Tada T, Yeo W, Mo F, Bettinger D, et al: Transarterial chemo-embolisation of hepatocellular carcinoma: Impact of liver function and vascular invasion. Br J Cancer 116: 448-454, 2017.

31. Bettinger D, Spode R, Glaser N, Buettner N, Boettler T, Neumann-Haefelin C, Brunner TB, Gkika E, Maruschke L, Thimme R and Schultheiss M: Survival benefit of transarterial chemoembolization in patients with metastatic hepatocellular carcinoma: A single center experience. BMC Gastroenterol 17: 98, 2017.

32. Dodson M, Redmann M, Rajasekaran NS, Darley-Usmar V and Zhang J: KEAP1-NRF2 signalling and autophagy in protection against oxidative and reductive proteotoxicity. Biochem J 469: 347-355, 2015.

33. Zhou Y, Wang Y, Wang X, Tian X, Zhang S, Yang F, Guo H, Fan R, Feng N, Jia M, et al: The protective effects of K-opioid receptor stimulation in hypoxic pulmonary hypertension involve inhibition of autophagy through the AMPK-MTOR pathway. Cell Physiol Biochem 44: 1965-1979, 2017.

34. Endo S, Nakata K, Ohuchida K, Takesue S, Nakayama H, Abe T, Koikawa K, Okumura T, Sada M, Horioka K, et al: Autophagy is required for activation of pancreatic stellate cells, associated with pancreatic cancer progression and promotes growth of pancreatic tumors in mice. Gastroenterology 152: 1492-1506 e24, 2017.

35. Auberger P and Puissant A: Autophagy, a key mechanism of oncogenesis and resistance in leukemia. Blood 129: 547-552, 2017.

36. Levy JMM, Towers CG and Thorburn A: Targeting autophagy in cancer. Nat Rev Cancer 17: 528-542, 2017.

37. Chen R, Wang H, Liang B, Liu G, Tang M, Jia R, Fan X, Jing W, Zhou X, Wang H, et al: Downregulation of ASPP2 improves hepatocellular carcinoma cells survival via promoting BECN1-dependent autophagy initiation. Cell Death Dis 7: e2512, 2016.

This work is licensed under a Creative Commons Attribution-NonCommercial-NoDerivatives 4.0 International (CC BY-NC-ND 4.0) License. 